\title{
THE IMPACT OF THE REVOLUTION OF DIAGNOSIS RADIOLOGY DEVICES ON THE HOSPITALS DESIGN
}

\section{ENG. Ahmed Mohammed Abdou}

Tutor Department of

Architecture,

Faculty of Engineering, Alazher University, Qena.

\section{Prof.Dr. Mohamed Abd- Elsamee Eid}

Professor of Architecture

The dean of

Faculty of Engineering, Assiut University
Dr. Nady Mostafa AbdelKarim

Associate prof. in Architecture Department, Faculty of Engineering Assiut University

(Received Journey 24, 2011 Accepted March 26, 2011)

Rays unit "rays department" is one of the most important units that is indispensable in hospitals. The diagnosis of a lot of diseases depends on this unit. More than a hundred years since the discovery of X-rays, the use of $X$ rays has developed. Some of these rays have been used in the field of treatment. Others have been used in the field of diagnosis.

Because the $X$-rays devices of diagnosis have greatly developed within very short time some confusion occurred in vacuum of rays department and other vacuums in the departments nearby. These devices have a remarkable effect on the role of hospitals on both service "diagnosis" and architecture. there are amendments and changes in the department of rays from time to time due to inventing new devices or transferring some devices towards non-centralization. This is done for the benefit of other departments of hospital, unless there is a strategic vision to deal with.

This paper aims at registering the great development of diagnostic rays and its architectural effect on the design of hospital and setting up a vision to deal with this case.

The research has proved that there is a change in the method of diagnostic services which change the department non-centralization. Besides some vacuums have become unwanted because they became useless after developing the devices of rays.

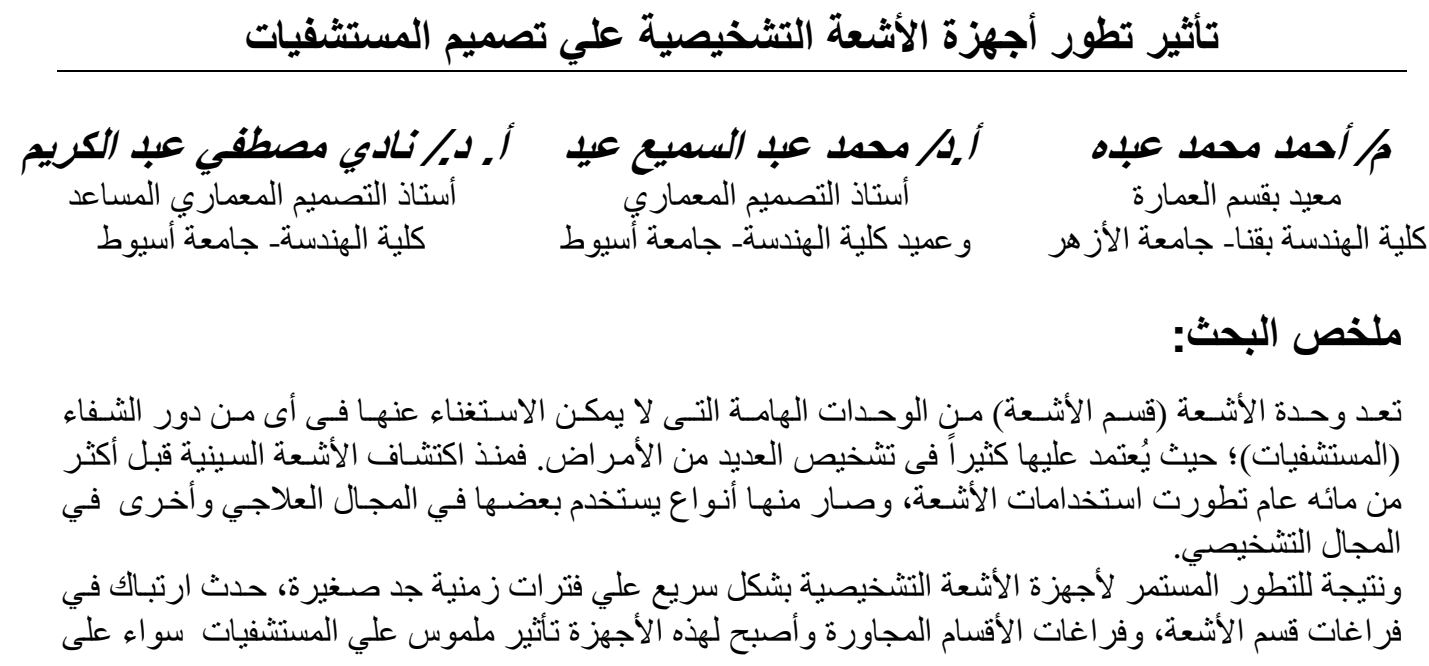




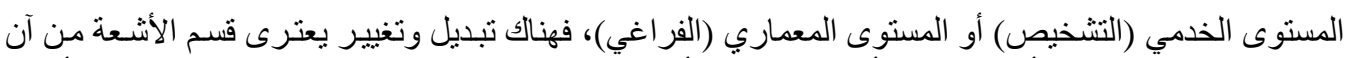

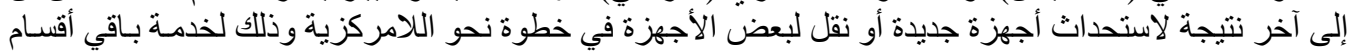
المنتشفى. ويهذف هذه. البحث إلى رصد التطور المستمر لأجهزة الأشعة التشخيصية، ومدى تأثير هـا فر اغياً على تصميم الكستشفى ووضع رؤية للتعامل مع هذه الحالة.

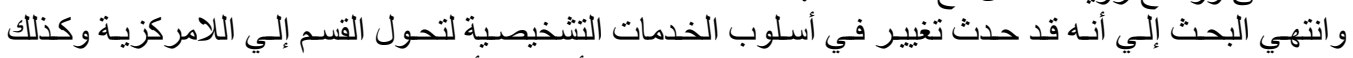
الاستغناء عن بعض الفر النهات التي لم بعد لها استخدام بعد تطور أجهزة الأشعة.

\section{1- 1 مقدمة البحث}

فى عام 1895م اكتثف العالم الألماني "رونتجن" أثشعة اكس، حيث أعلن في نهاية العام عن إمكانية تطبيق هذا

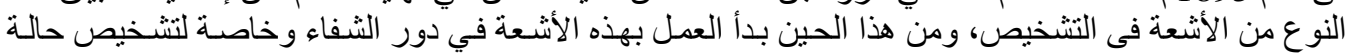

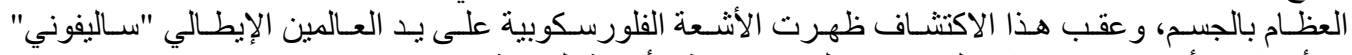

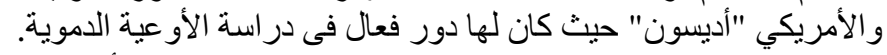

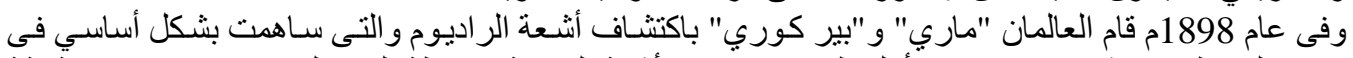

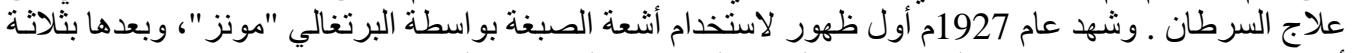

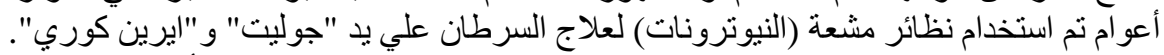

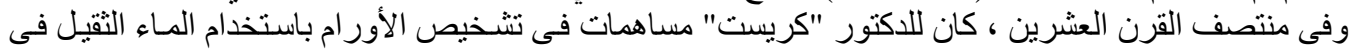

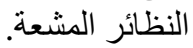
وفى نهاية القرن العشرين تم إدخال الجيل الأول من أجهزة التشخيص الالكترونية (باستخدام الكمبيوتر ) وأهم

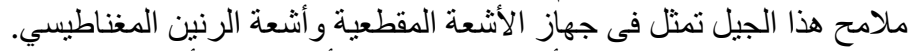

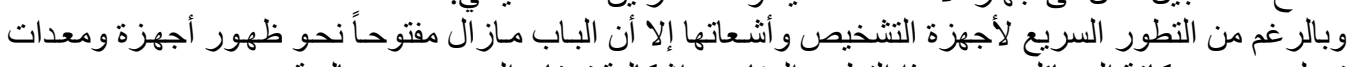

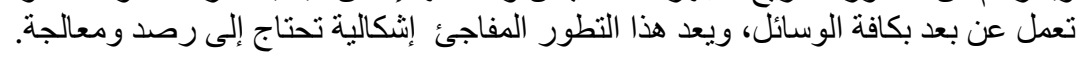

\section{2- 2 - إثكالية البحث}

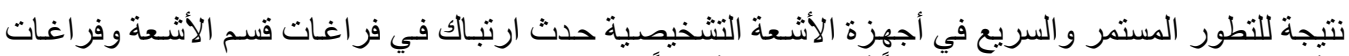

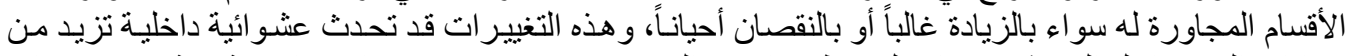

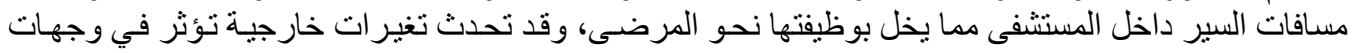
المستشفي مما يخل بجماليات العمارة.

\section{3- منهجية البحث وهدفه}

ـ اتبع البحث المنهج التحليلي من خلال الآتي:

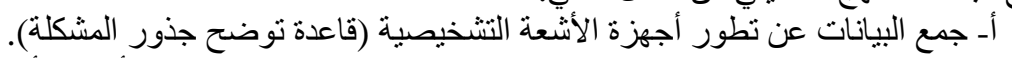

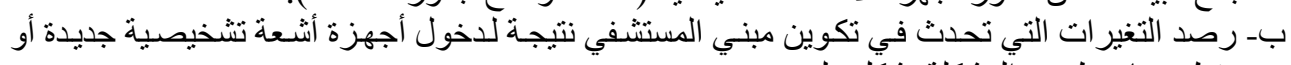

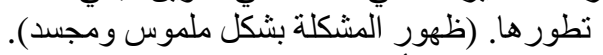

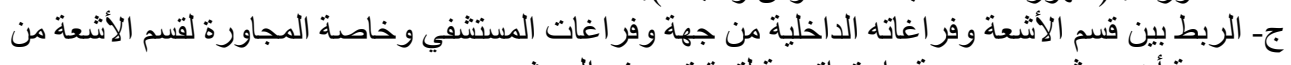

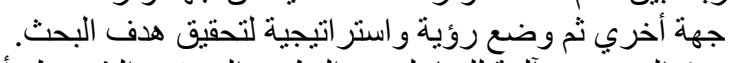

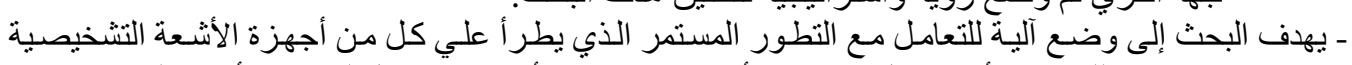

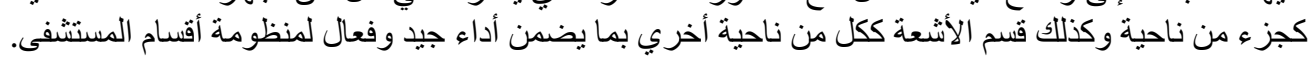

\section{4- التطور التاريخي لأجهزة الأشعة التثخيصية}

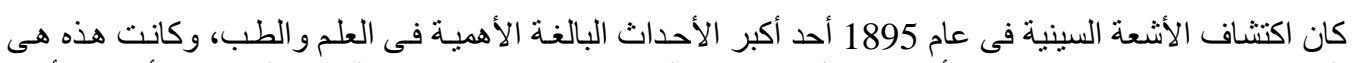

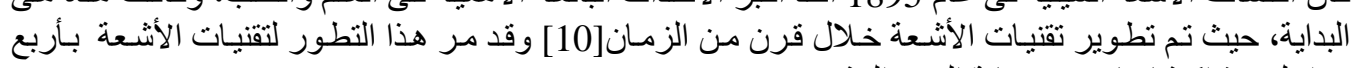
مر احل منذ اكتثافها وحتى نهاية القرن العشرين وهى: 


\section{1-4 المرطلة الأولى: نهاية القرن التاسع عشر (1895-1900):}

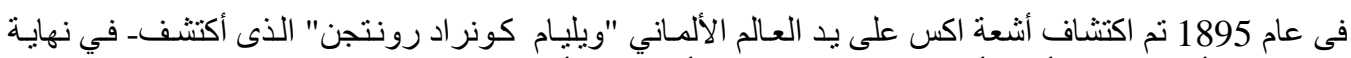

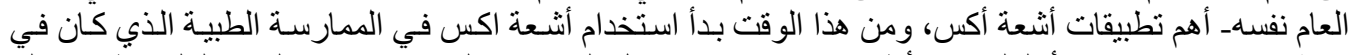

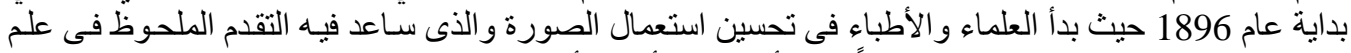

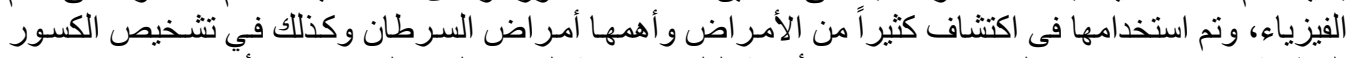

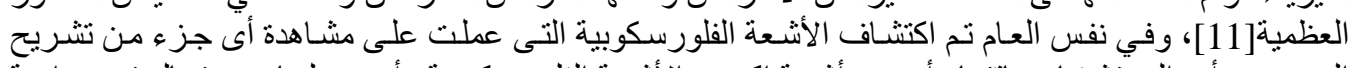

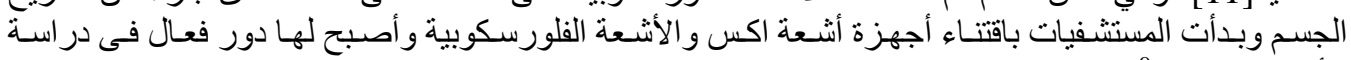

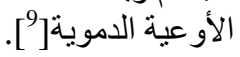

\section{2-4 المرحلة الثانية: مرحلة باية القرن العشرين(1900-1940):}

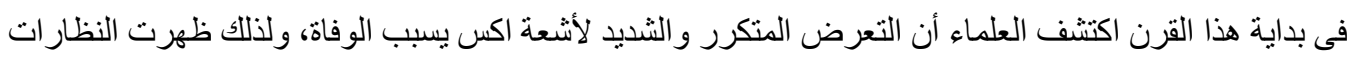

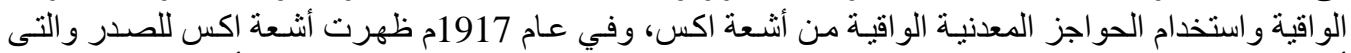

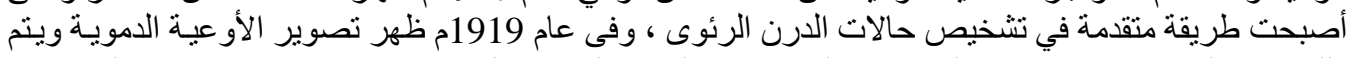

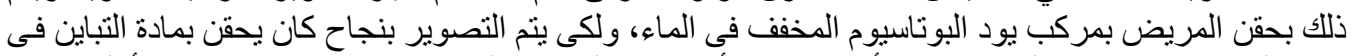

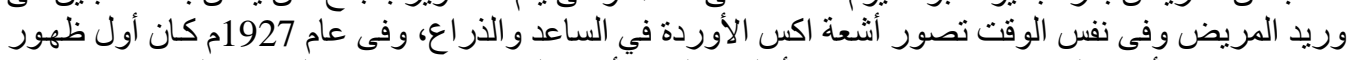

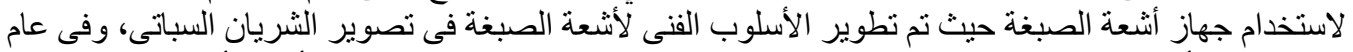

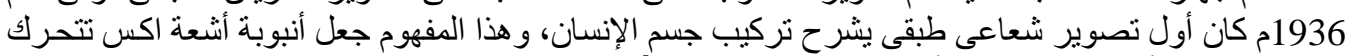

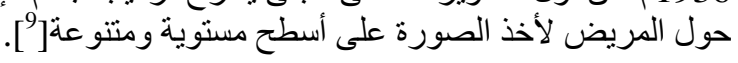

3-4 المرحلة الثالثة: مرحلة منتصف القرن العشرين(1940-1970):

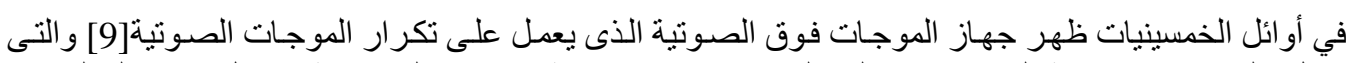

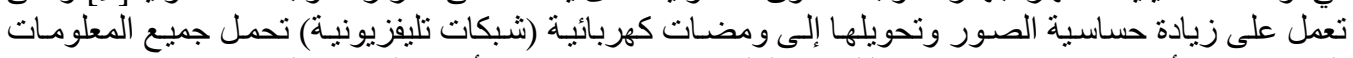

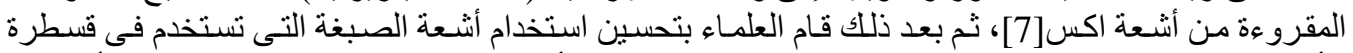

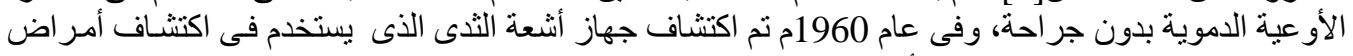

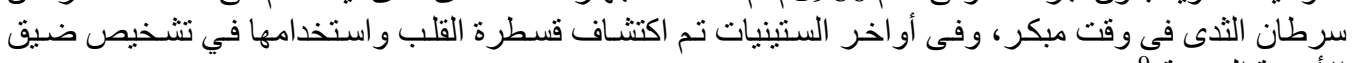

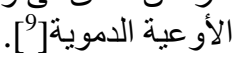

\section{4-4 المرحلة الرابعة: مرحلة نهاية القرن العشرين(1970-2000):}

فى بدايـة السبعينيات تم إدخال الجيل الأول من أجهزة التشخيص باستخدام الكمبيونز والتى تتمثل في الأشـعة

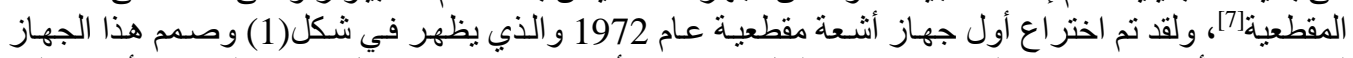

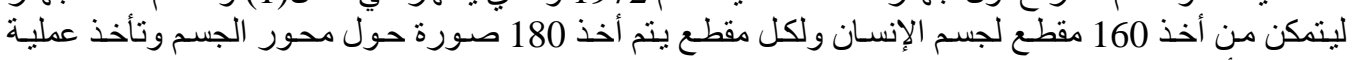

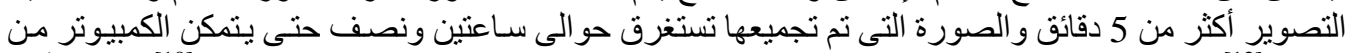

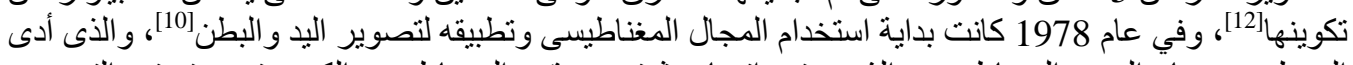

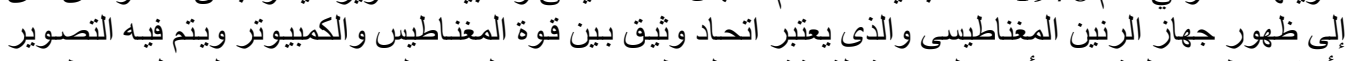

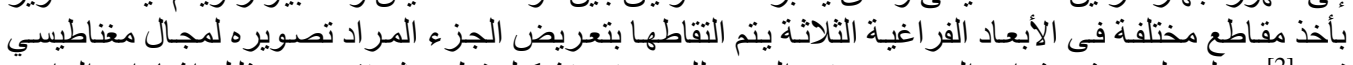

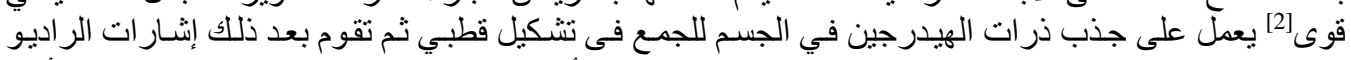

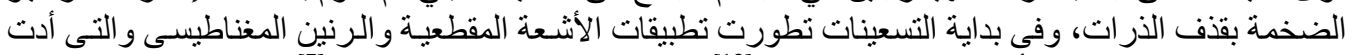

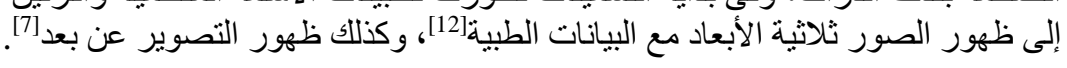

\section{5- التطور التكنولوجى لأجهزة الأشعة التشخيصية بعد الألفية الثاتية:}

فى السنوات الأخيرة ومع وجود تقنيات متطورة في مجال صناعة الأجهزة فـان اتجاهـات الاندماج مـع التقنيات

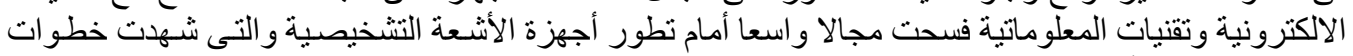

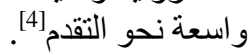




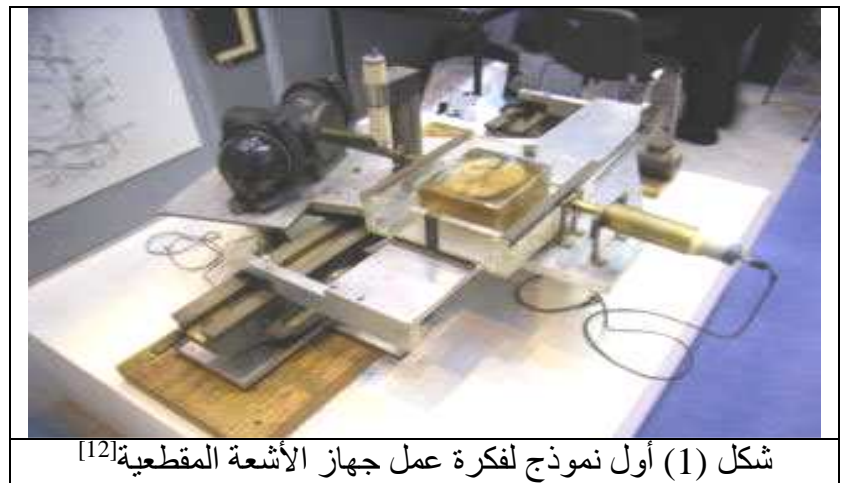

1-5 ظهور أجيال جديدة من أجهزة الأشعة التثخيصية:

أدت المستجدات العلمية فى مجالات الفحوص الإشعاعية والتشخيصية فى مختلف المجالات الطبية فى المسنشفيات

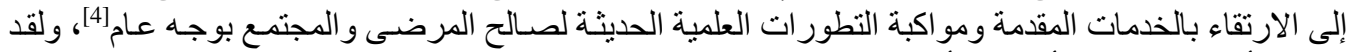

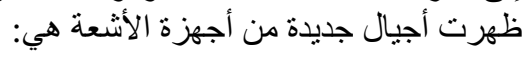

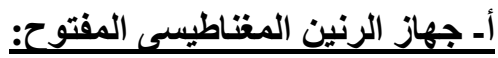

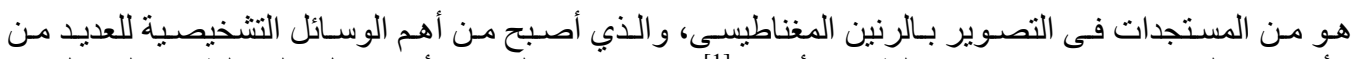

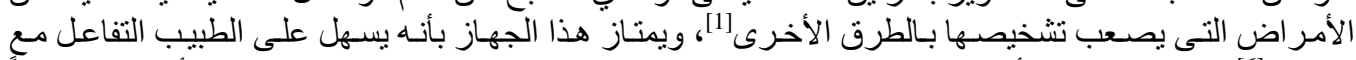

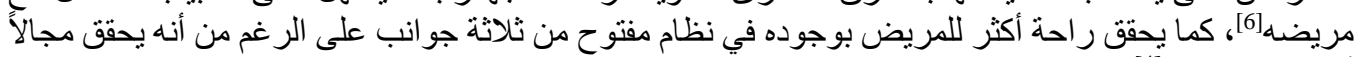

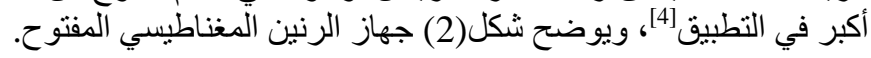

\section{بـ بـ أجهزة Allura Xper}

يمتلك هذا الجهاز تخصصاً عالياً في عمل الصور ثلاثية الأبعاد، ويستخدم هذا الجهاز أخر المفاهيم والتكنولوجيا

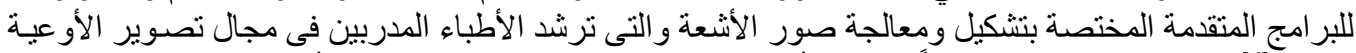

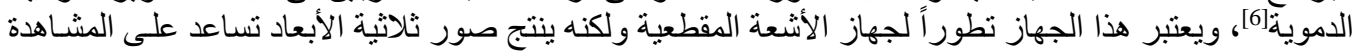

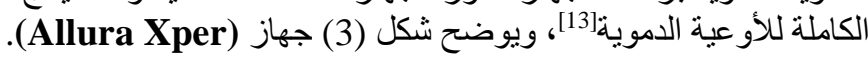

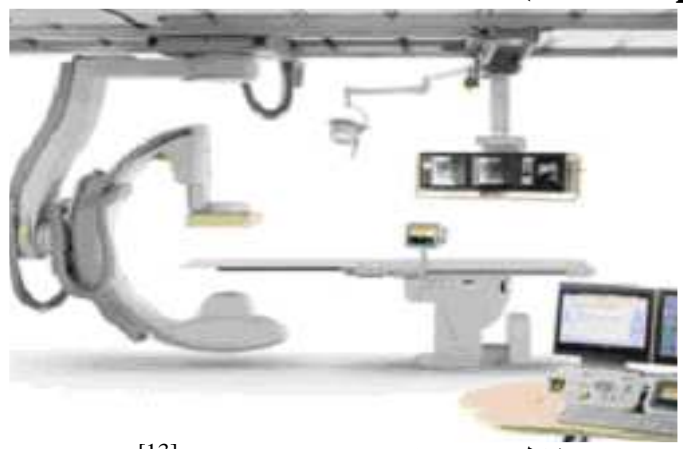

شكل(3) جهاز (Allura Xper)

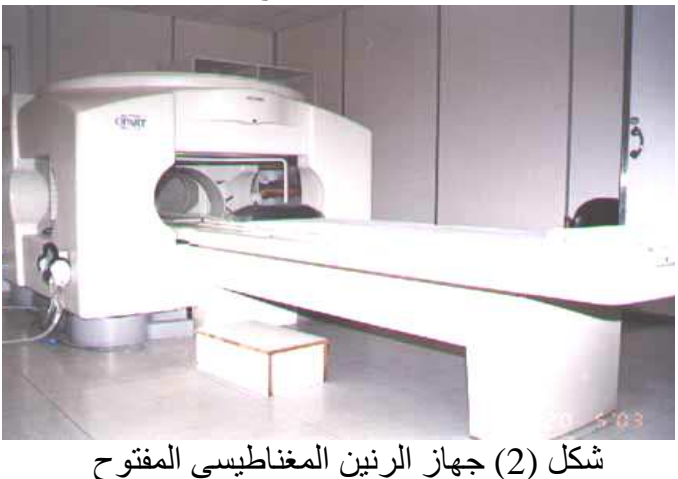

ج- جهاز الموجات فوق الصوتية رباعى الأبعاد:

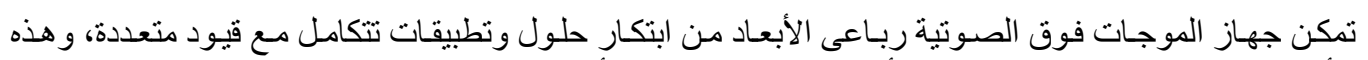

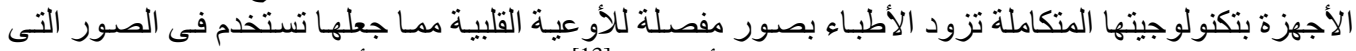

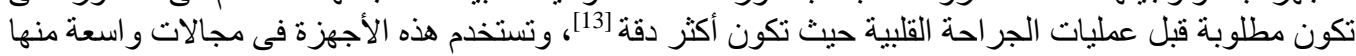

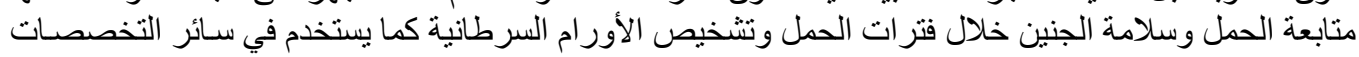


الطبية حيث توجد فى أقسام المسالك البولية والكلى و أقسام الأمر اض الباطنيـة والقلب و الصدر و الهـخ والأعصـاب و أقسام العيون.

\section{دـ جهاز الأشعة المقطعية المبرمج:}

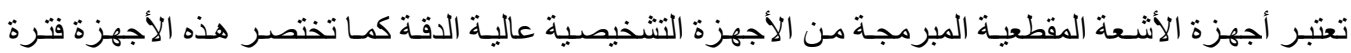

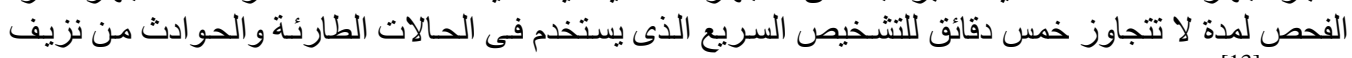
وكسور [13.

\section{هـ جهاز التصويز المحورى الطبقى:}

من أبرز التطور ات التى حدثت فى مجال الطب فى بداية القرن الو احد و العثرين قيام احدى الشركات في مجال

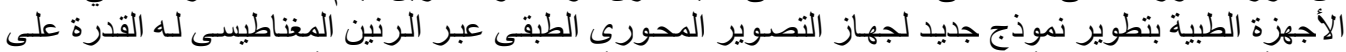

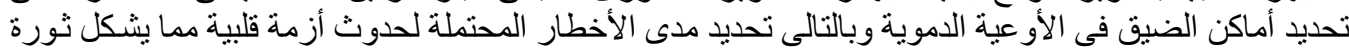

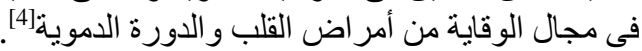

\section{6- أثر تطور أجهزة الأشعة التشخيصية على تصميم المستثفى:}

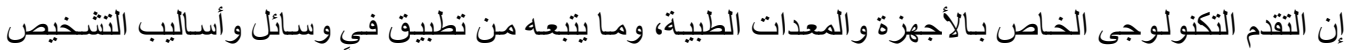

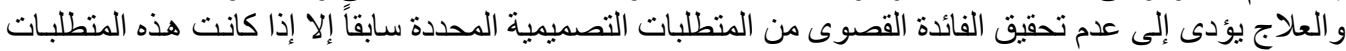

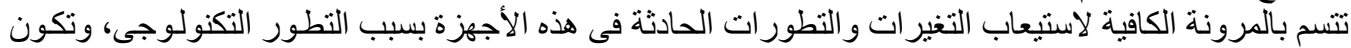

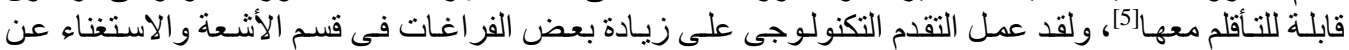

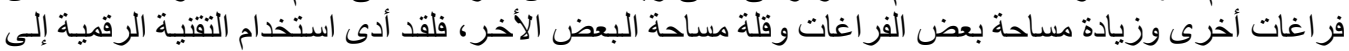

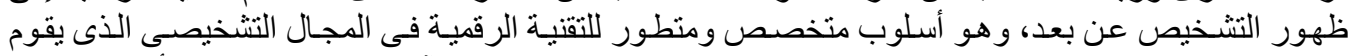

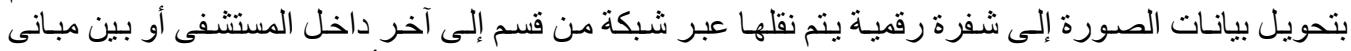

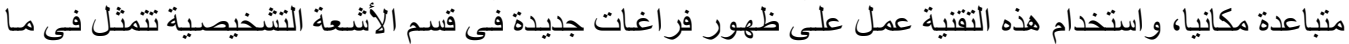
يلى [2]:

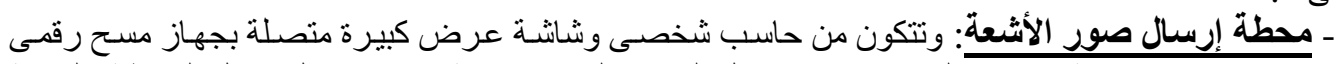

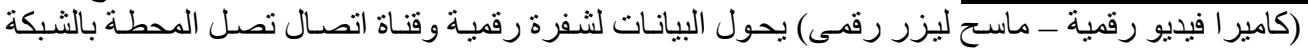
و ومنها إلى محطة الاستقبال.

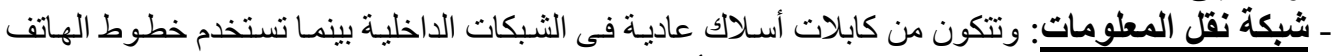

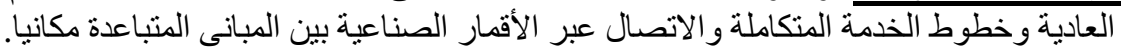

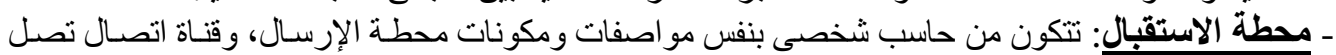

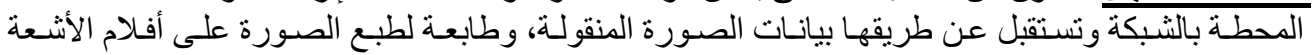

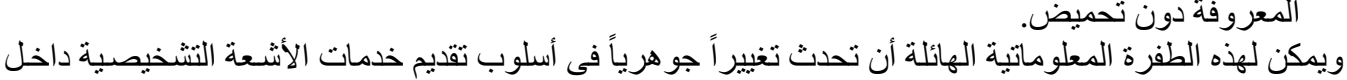

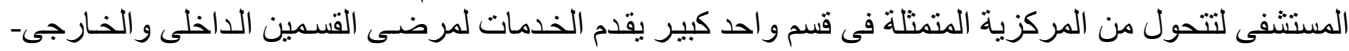

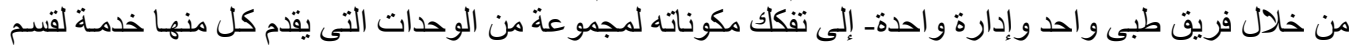

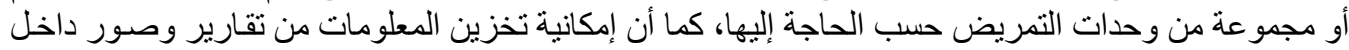

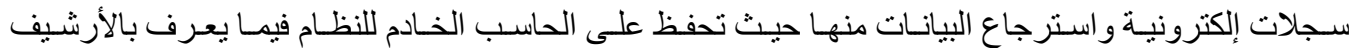

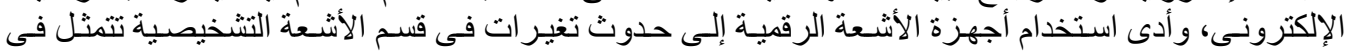

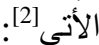
ـ توسع كيفى فى منطقة السكرتارية ومنطقة كتابة التقارير الطبية، حيث تحولت كل منها إلى فر اغ أكثر اتسـاعا

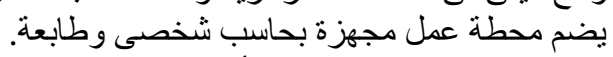

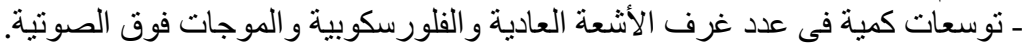

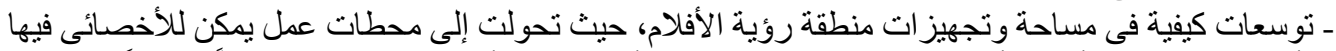

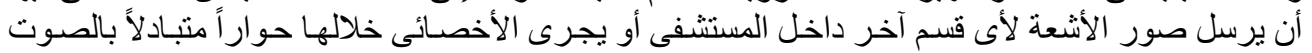


و الصورة مع الطبيب الجر اح عن طريق كامير ات فيديو رقميـة وشاثـة عرض لمناقشته فى صـورة الأشـعة المأخوذة للمريض أثناء العملية.

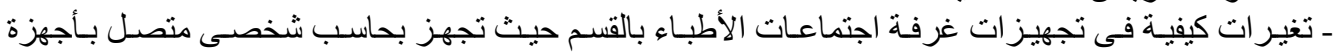

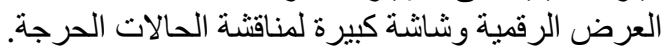

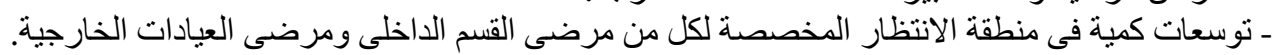

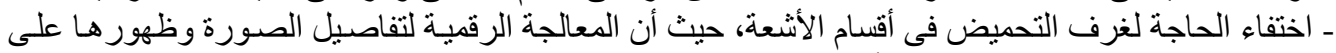

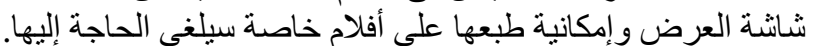

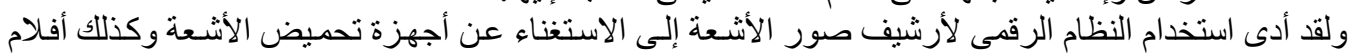

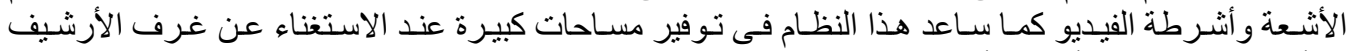

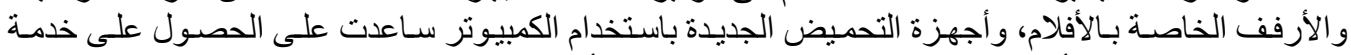

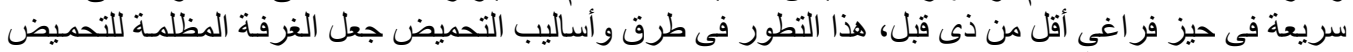

من منطلبات الماضىى [5 فئ. و التقدم التكنولوجى في أجهزة الأشعة التشخيصية يؤثر على كثير من أقسام المستشفى حيث توجد عدة أقسـام

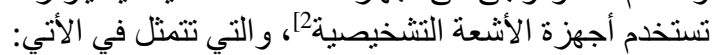

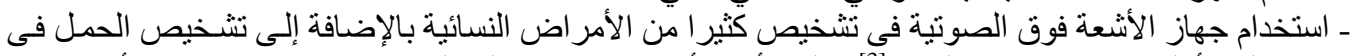

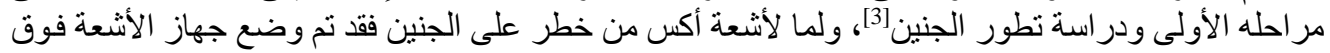
الصوتية فى قسم النساء و الو لادة.

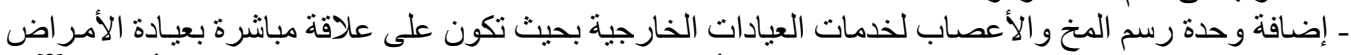

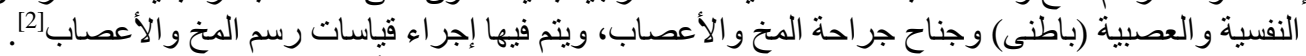

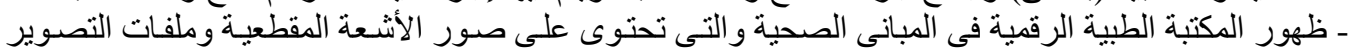

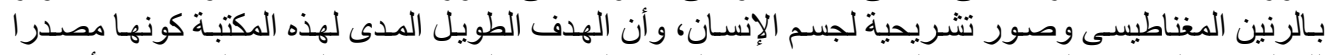

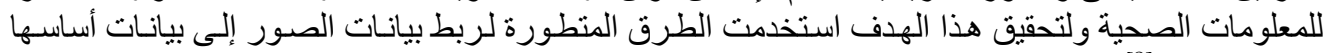
النص الرمزى [8]ت.

\section{7- تأثير تطور أجهزة الأشعة علي تصميم مستثفي قائم}

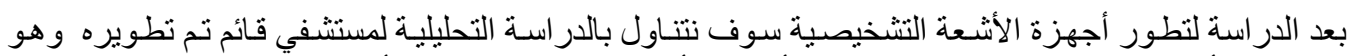

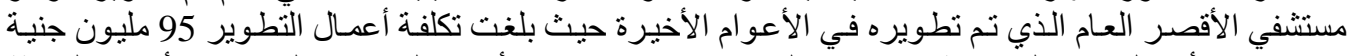

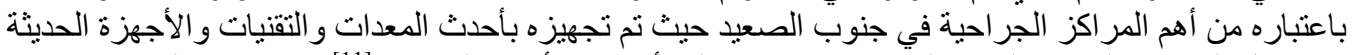

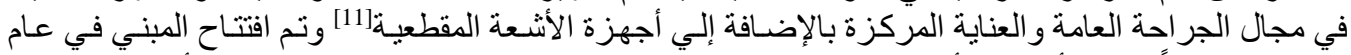
2010م، ونظر اً لتطور أجهزة الأشعة التشخيصية حدثت تطور التئ ملحوظة في تصميم المسقط الأفقي للمستشفي تتمثل في الآتي:

\section{1-7 زيادة مساحة قسم الأثُعة التثخيصية في المستثفي:}

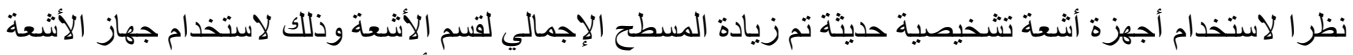

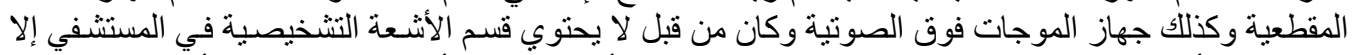

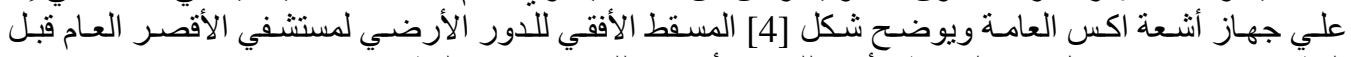

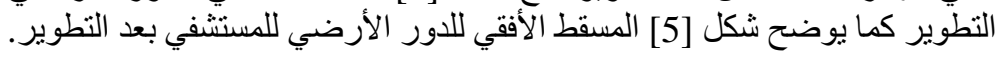

\section{2-7 الاستغناء عن بعض فراغات قسم الأشعة:}

نظر التطور قسم الأشعة واعتماد أكثر الأجهزة علي التقنيات الرقمية تم الاستغناء عن بعض فر اغات قسم الأشعة

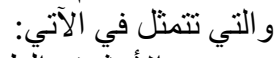

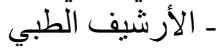

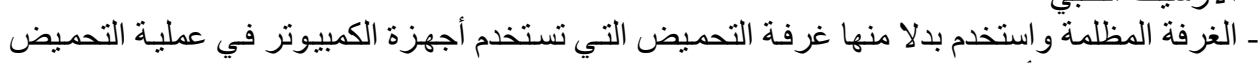
و عدم استخدام الأصباغ المستخدمة فيما قبل. 
ويوضح شكل [6] المسقط الأفقي لقسم الأشعة قبل التطوير كمـا يوضـح شكل [7] المسقط الأفقي لقسم الأشـعة التشخيصية بعد التطوير .

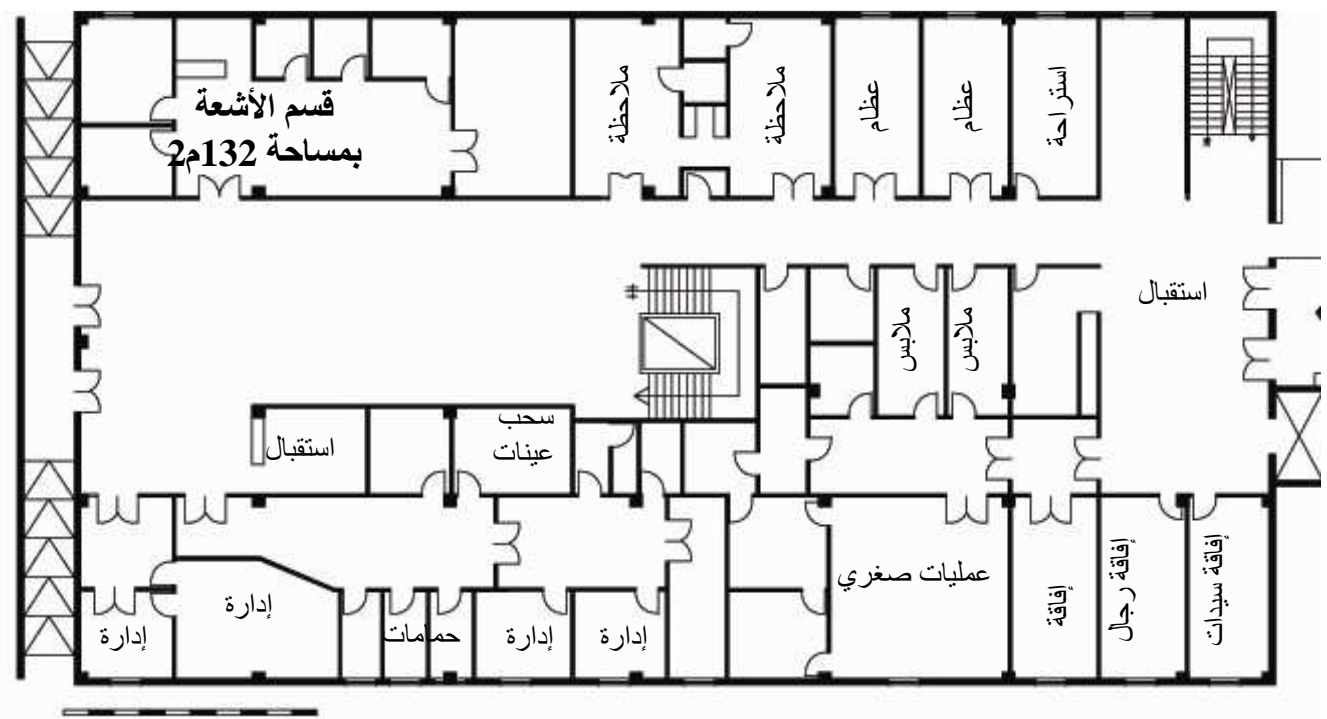

شكل[4] المسقط الأفقي للاور الأرضي لمستشفى الأقصر العام قبل التطوير

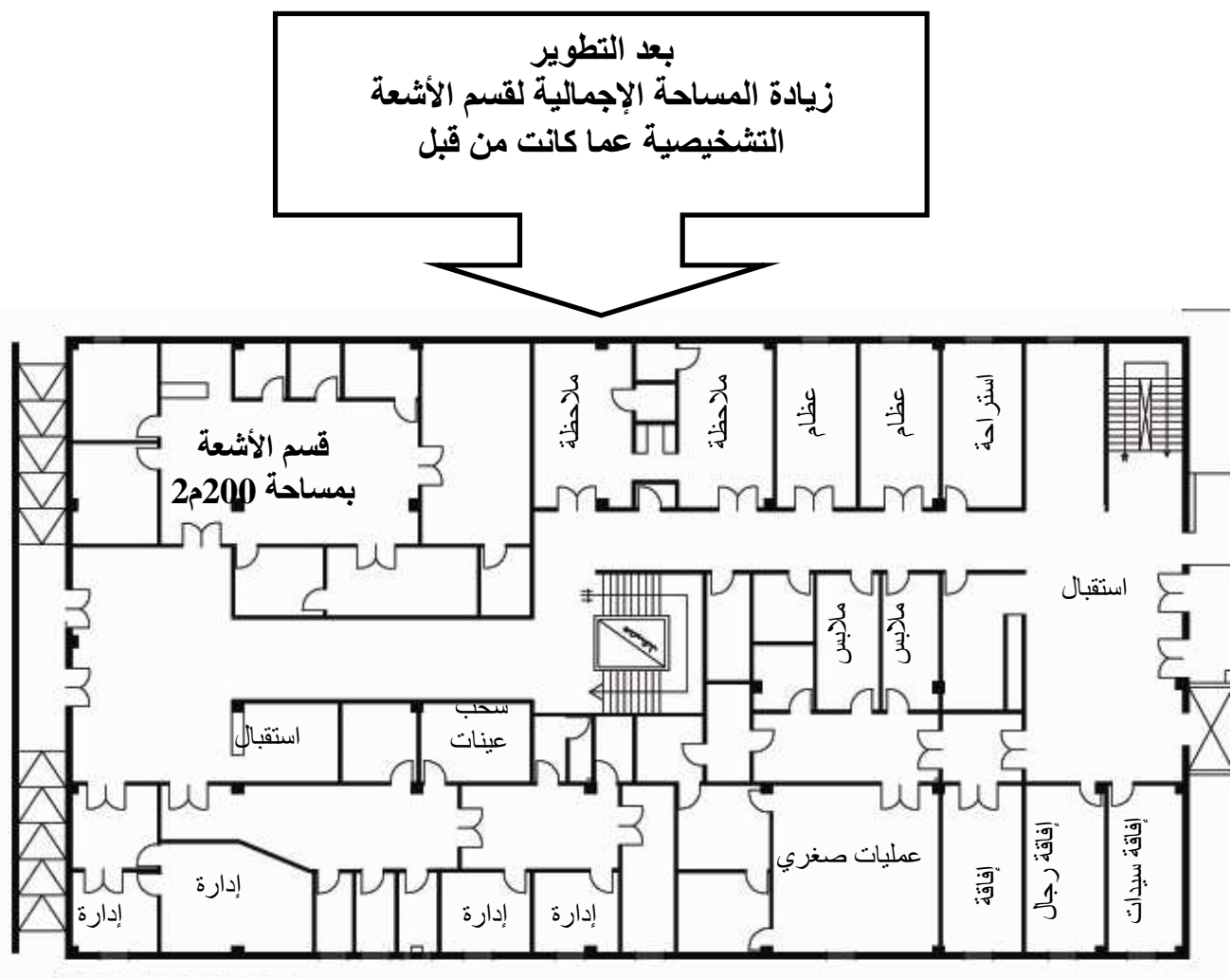

شكل[5] المسقط الأفقي للاور الأرضي لمستشفى الأقصر العام بعد التطوير 


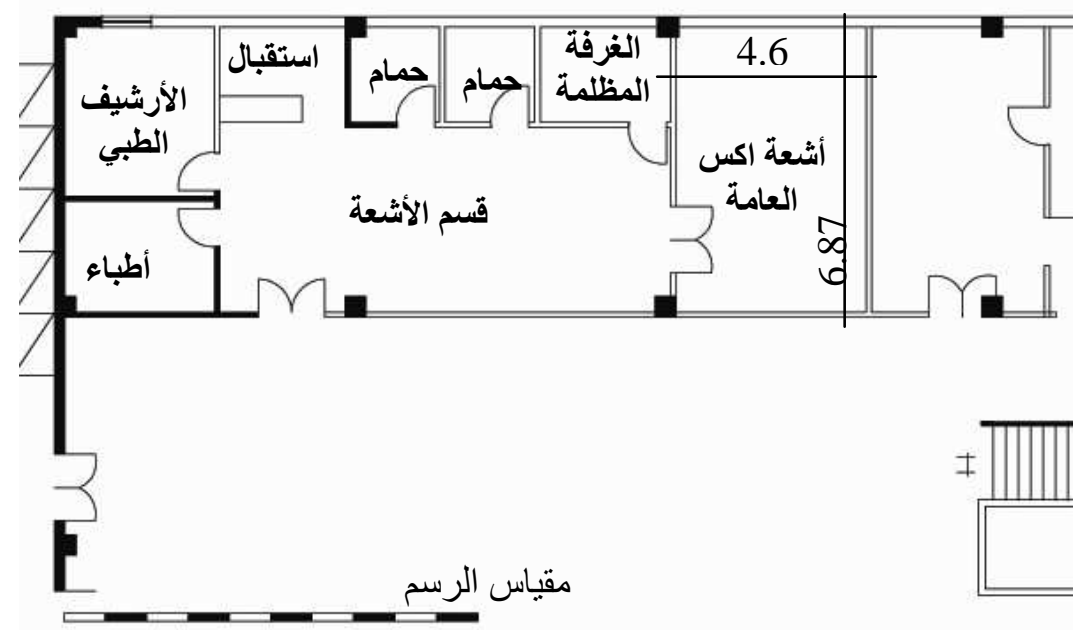

شكل [6] المسقط الأفقي لقسم الأشعة التشخيصية قبل التطوير

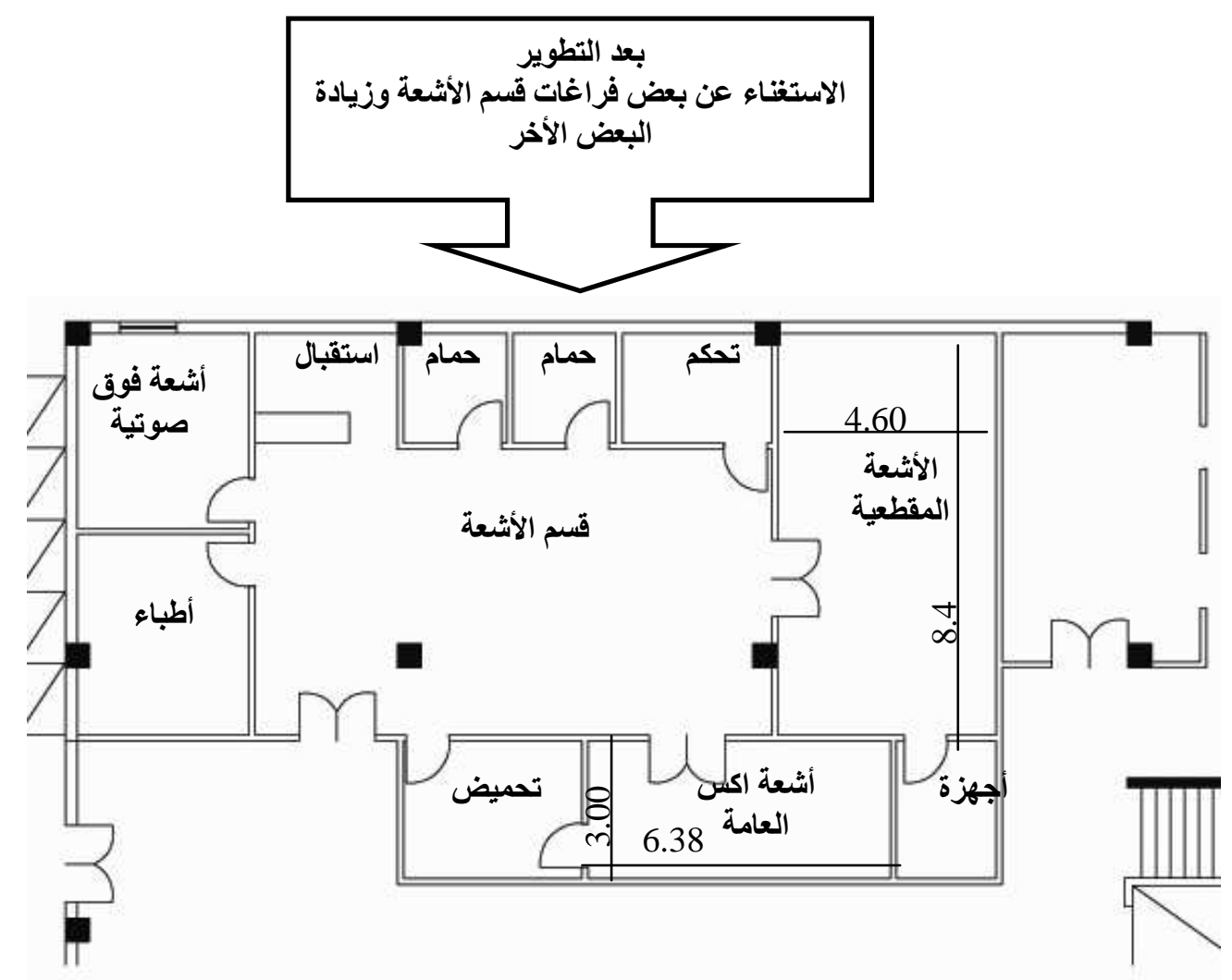

شكل [7] المسقط الأفقي لقسم الأشعة التشخيصية بعد التطوير 
3-7 تحول قسم الأثعة إلي اللامركزية :

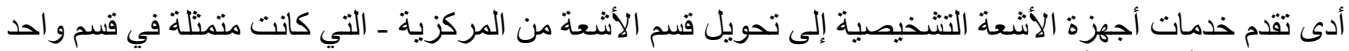

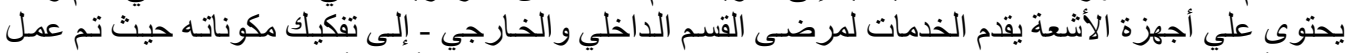

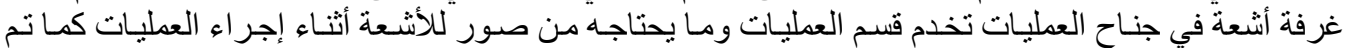

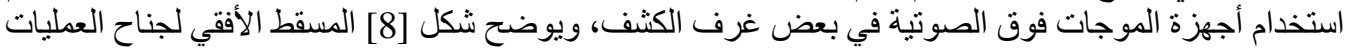
قبل التطوير ،كما يوضح شكل [9] المسقط الأفقي لجناح العمليات بعد التطوير.

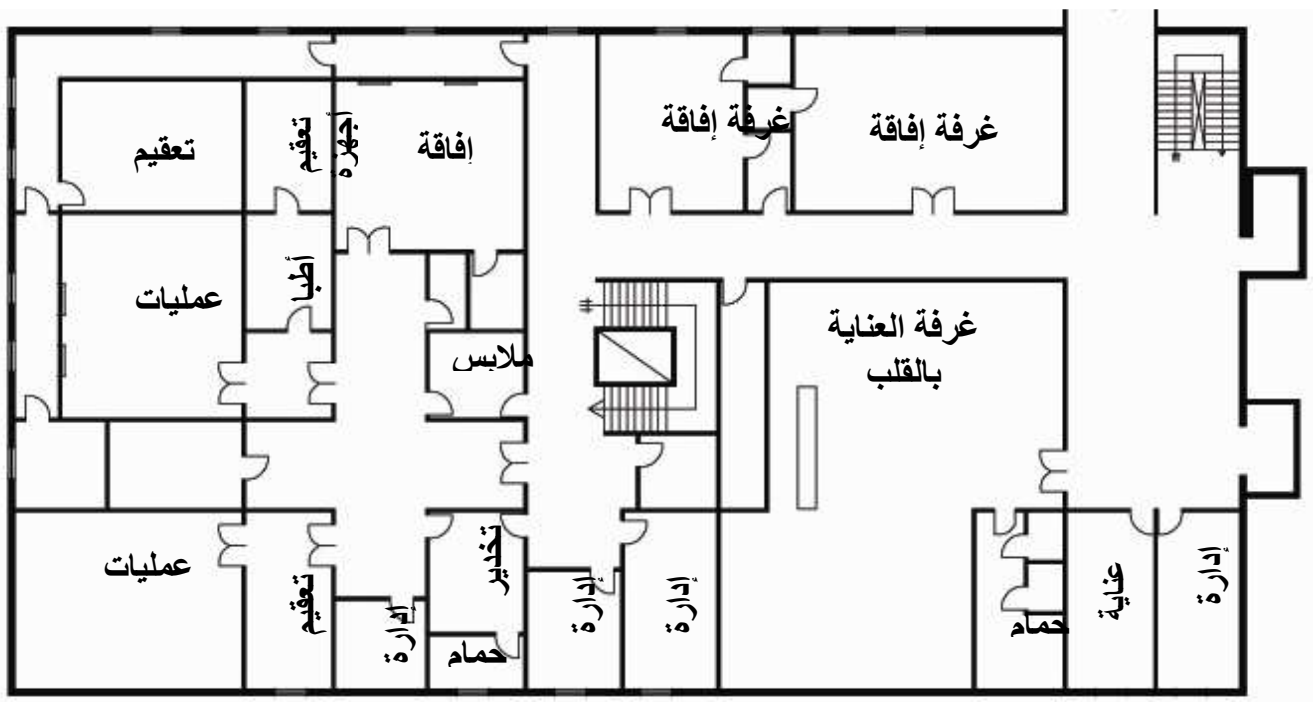

شكل [8] المسقط الأفقي لجناح العمليات قبل التطوير

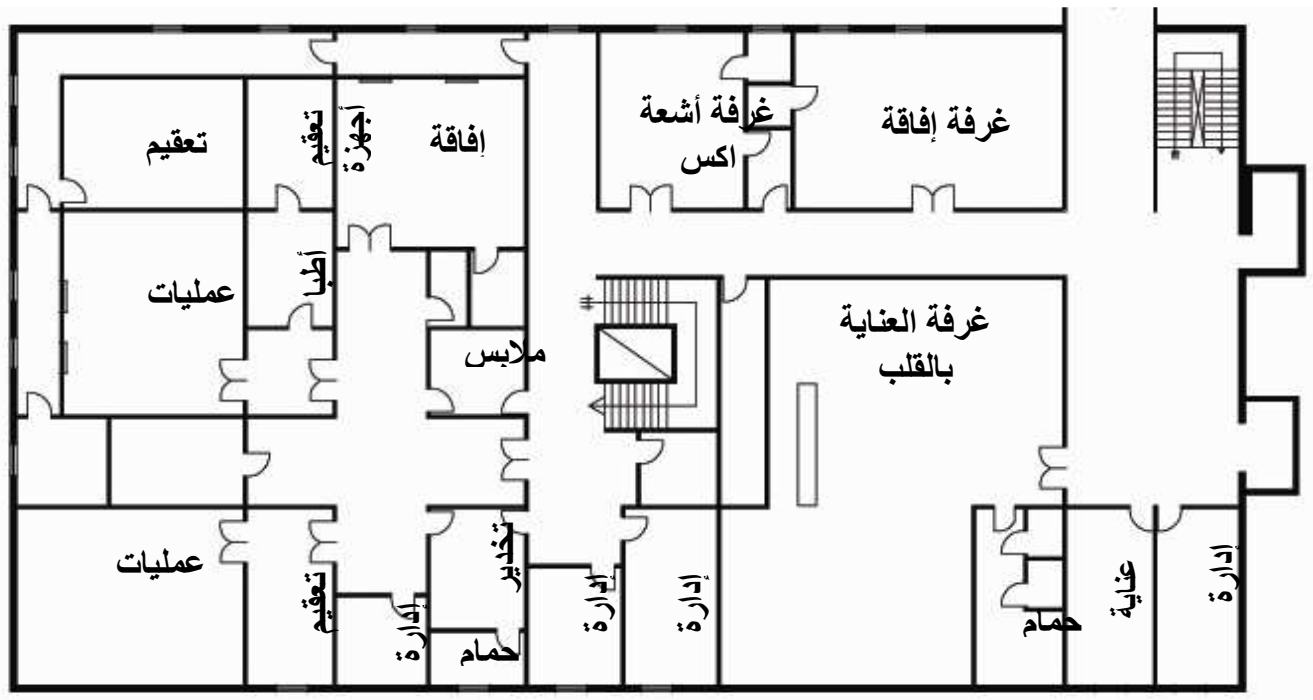

شكل [9] المسقط الأفقي لجناح العطليات بعد التطوير يحتوى على غرفة أشعة 


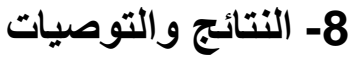

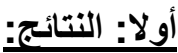

1- أدي استخدام التقنية الرقية في المجال التشخيصي إلي ظهور فر اغات جديدة في قسم الأشعة مثل محطة

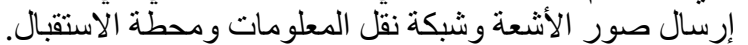

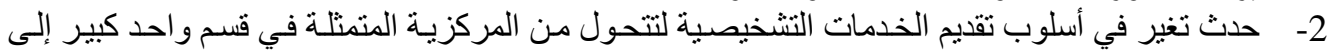

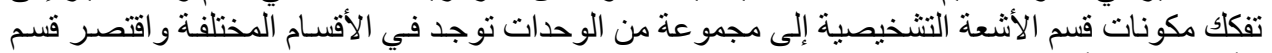

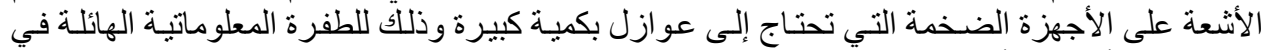
مجال تطور أجهزة الأشعة التشخيصية.

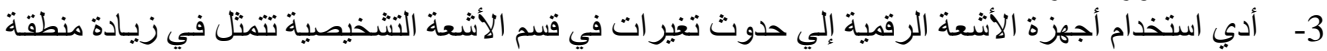
السكرتارية ومنطقة كتابة التقارير الطبية.

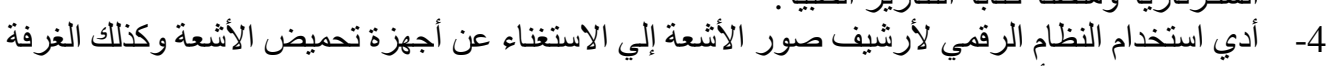

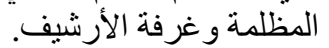
5- يادة المساحة الإجمالية لقسم الأثعة التشخيصية عما كان موجوداً من قبل وذلك لظهور أجهزة أثنعة حديثة ومتنو عة ومتطورة.

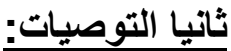

من النتائج السابقة يقترح البحث مجموعة من التوصيات يفضل استخدامها عند البدأ في تصميم قسم الأشعة

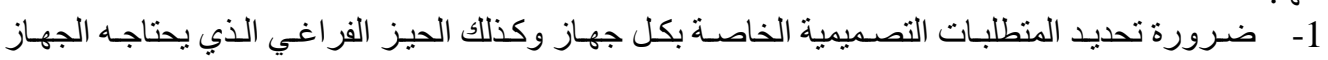
التركيبه وتثنغيله وصياتته قبل البدء في عملية التصمية..

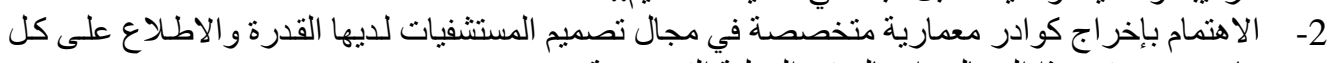
ما هو جديد في هذا المجال و إدخاله في العملية التصميمية.

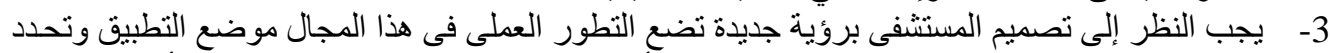

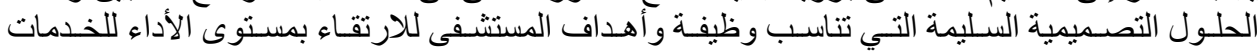

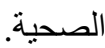

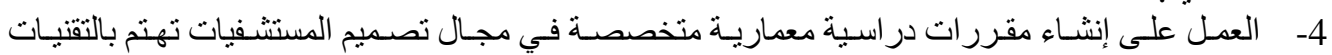

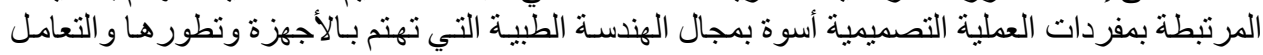
معهاوصيانتها. - مبردات

\section{المراجع}

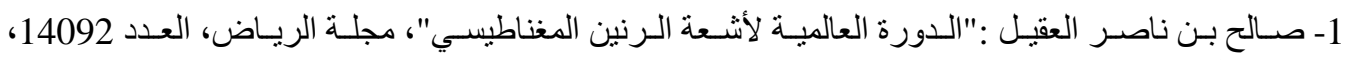
23 2007 2007م.

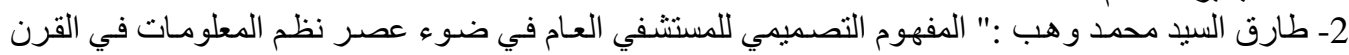

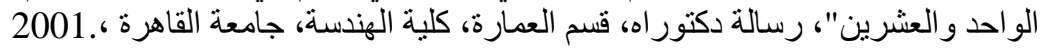

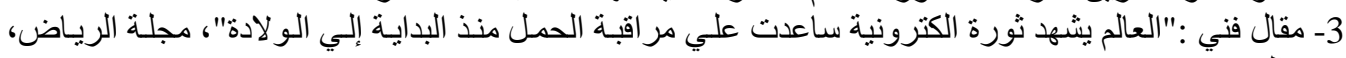

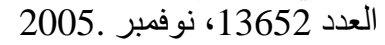

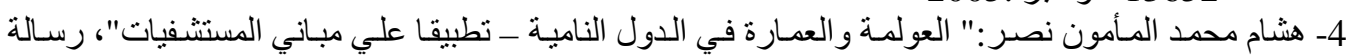

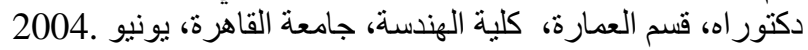

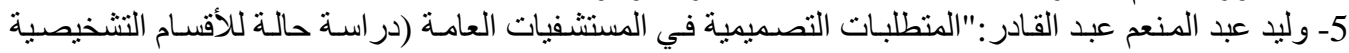

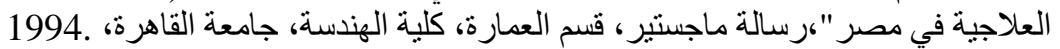

6- Cardiovas Cular Research:"Physician Training Center at TCT2006" interventional vascular simulation, the university hospital of columbia, columbia university, medical center New york, 22-27 octoper 2006 
7- Francesca Giofre :"Design for Health the Biomedical Equipments" , Master of second level in planning, planning and management of the sanitary building in the countries of the Mediterranean, Roma, 2004

8- M. Tsiknakis, and other:"An Integrated Architecture for the provision of Health Telematic Services Based on Digital Library Technologies " 1997.

9- Nady mostafa:" Diagnostic imaging ", Master of second level in planning, desiging and management of the health care buildings in the countries of the nediterraiam, Rome, 2004

10-otha.W.Elinton :"Medical applications of X-Ray", www.slac.Stanford.edu, summer 1995.

11 - www.ahram .org.eg (مجلة الأهر ام، العدد 45135، 4يوليو 2010)

12- www.hazemsakeek.com/QandA/x-ray/x-ray.htm (10/2006)

13-www.Medical.philips.com/ch-de/news/content/file,(KLAS Enterprises) (10/2008) 